Review Article

\title{
Systematic Review and Meta-Analysis of 16 Randomized Clinical Trials of Radix Astragali and Its Prescriptions for Diabetic Retinopathy
}

\author{
Lin Cheng, ${ }^{1}$ Gai Zhang, ${ }^{2}$ Yi Zhou, ${ }^{3}$ Xuejing Lu, ${ }^{2}$ Fuwen Zhang, ${ }^{2}$ \\ Hejiang Ye, ${ }^{2}$ and Junguo Duan ${ }^{2}$ \\ ${ }^{1}$ Institute of Clinical Pharmacology, Central South University, Changsha, Hunan 410078, China \\ ${ }^{2}$ National Diabetes Mellitus Clinical Research Base of TCM, Teaching Hospital of Chengdu University of TCM, \\ Chengdu, Sichuan 610075, China \\ ${ }^{3}$ College of Clinical Medicine, Chengdu University of TCM, Chengdu, Sichuan 610075, China
}

Correspondence should be addressed to Junguo Duan; duanjg@vip.sina.com

Received 20 March 2012; Accepted 4 January 2013

Academic Editor: Benny Tan Kwong Huat

Copyright (C) 2013 Lin Cheng et al. This is an open access article distributed under the Creative Commons Attribution License, which permits unrestricted use, distribution, and reproduction in any medium, provided the original work is properly cited.

\begin{abstract}
Objective. To evaluate the efficacy and safety of radix astragali and its prescriptions for diabetic retinopathy. Methods. A computerbased online and manual search was conducted for randomized controlled trials addressing radix astragali and its prescriptions for diabetic retinopathy. Results. 16 RCTs involving 977 subjects and 1586 eyes were identified. Meta-analysis indicated that the effect of radix astragali and its prescriptions in improving visual acuity and fundus manifestations, lowering FBG, TG, plasma viscosity, and RAI, was superior to that of control group (WMD or OR $0.20,0.27,-0.26,-0.36,-0.93,-1.27 ; 95 \% \mathrm{CI}[0.09,0.30],[0.17,0.40]$, [-0.51, $0.00],[-0.60,-0.12],[-1.67,-0.20],[-2.35,-0.19] ; P<0.05$, resp.). In contrary, the efficacy of radix astragali and its prescriptions was not superior to those of control group in descending HbA1C and TC with WMD 0.45, -0.96 and 95\% CI [-1.00, 1.90], [-2.19, 0.27], $P>0.05$, respectively. GRADE software suggested that the studies were of low methodological quality. Conclusion. Radix astragali and its prescriptions were superior to other treatments for diabetic retinopathy in terms of improving visual acuity and fundus manifestations, reducing FBG, TG, RAI, and plasma viscosity. The evaluated studies were of low methodological quality, indicating that the previous findings should be read with care.
\end{abstract}

\section{Introduction}

Diabetic retinopathy (DR) is a disease of retina as a complication of diabetes mellitus, resulting in loss of vision, macular edema, recurrent vitreous hemorrhages, tractional or secondary rhegmatogenous retinal detachment, and so forth [1-5]. It is characterized by the progressive microvascular complications, such as aneurysm, intraretinal edema, and intraocular pathologic neovascularization [6]. It is an accepted fact that diabetic retinopathy does not occur for at least 2-5 years after the onset of Type 1 (insulin-dependent) or type 2 (non-insulin-dependent) diabetes mellitus [7, 8]. Diabetic retinopathy is not only one of the main microvascular complications of diabetes, but also an important public health problem $[9,10]$. Approximately 2.5 million people worldwide are clinically blind attributed to diabetic retinopathy [11]. In particular, DR remains a major threat to eyesight in the working age population $[12,13]$. The available data suggest that the global number of people with DR will grow from 126.6 million in 2010 to 191.0 million by 2030, among them vision-threatening DR is estimated to increase from 37.3 million to 56.3 million [14]. As the incidence of diabetes gradually increases, there is the possibility that more individuals will suffer from eye complications, which, if not properly managed, may lead to permanent eye damage.

Diabetic retinopathy can be managed by improved control of glucose and blood pressure $[15,16]$, pharmacological, laser, and surgical approaches [17, 18]. Principal pharmacological therapies include drugs that inhibit neovascularization, such as anti-VEGF derivatives (Avastin \& 
Lucentis [19-21]), or drugs to relieve macular swelling such as steroidal anti-inflammatory drugs (triamcinolone acetonide [22]). Although the laser treatment with or without antiVEGF drugs is considered the "Golden Standard" treatment of diabetic retinopathy, it is only suitable for high risk of proliferative DR or proliferative DR. Laser and surgical interventions can be very effective for diabetic retinopathy but, as yet, final choices when it draws near to proliferative diabetic retinopathy. Would there be some therapies that can prevent the progression of DR? With the distinctive traditional medical opinions and natural medicines mainly originated in herbs, the traditional Chinese medicine performed a good clinical practice and is showing a bright future in the therapy of diabetes mellitus and its complications [23]. In treating wasting-thirst/diabetes and its complications, Traditional Chinese Medicine (TCM) harbors a long history, and herb treatment is also various. Zhang et al. have done research by mining and reviewing the literature in the 2000-year history of wasting-thirst and suggested TCM has a profound understanding and effective management on diabetes and its complications [24]. Some scholars had statistical analysis of ancient Chinese treating formulas and found that radix astragali (Chinese medical herb, also named huang qi) was the most popular herb to treat diabetes [25, 26]. As in the ancient days, wasting-thirst/diabetes and its complications were treated together according to syndrome differentiation. This suggested that radix astragali was one of the most common used herb for DR. Up till now, there is insufficient evidence to support the efficacy of radix astragali and its prescriptions for diabetic retinopathy. Due to its extensive application, the authors insisted that it is necessary to do a systematic review to explore radix astragali and its prescriptions in treating diabetic retinopathy.

This study was designed to assess the efficacy and safety of radix astragali and its prescriptions in treating diabetic retinopathy by conducting literature reviews in Chinese and English databases for RCTs. In this systematic review and meta-analysis, multiple publications reporting the same group of participants, or their subsets, were excluded.

\section{Data and Methods}

2.1. Search Strategy. The following sources were searched up to Septemper 2010: The Cochrane Library, Medline, EMBASE, Current Controlled Trials, Chinese Biomedical Literature Database (CBM), China National Knowledge Infrastructure (CNKI), VIP database, Wanfang database, 1980-2010.06 inclusive. We also conducted manual search of relevant journals, symposia, degree papers, and conference proceedings to retrieve relevant trials for cross checking some data that may be missed on electronic devices. The reference lists of papers were checked for further relevant publications, and experts were asked for information concerning any additional trials. Personal contact was made with the authors of the studies, if necessary, to request for additional data.

Search terms used to search RCTs were ("diabetic retinopathy" OR "eyeground changes" OR "fundus disease" OR "fundus changes" OR "eye disease") AND ("herb" OR "radix astragali" OR “astragalus mongholicus" OR "astragalus membranaceus" OR “astragalus” OR "huangqi”) AND ("randomized controlled trial" OR "controlled clinical trial” OR "random” OR "randomly” OR “randomized” OR “control”).

\subsection{Inclusion and Exclusion Criteria}

2.2.1. Type of Studies. Our paper was restricted to RCTs that compare radix astragali and its prescriptions for diabetic retinopathy to a control group, including placebo, no treatment (blank), or conventional treatment (western medicine treatment), other herb treatment (except radix astragali)) but not including laser treatment and acupuncture. Observational studies, reviews, animal studies, nonrandom or quasirandom studies, studies not taken visual acuity, as one of outcome measures or the description of visual acuity was not clear enough for statistics, were excluded. No restriction was imposed on studies with respect to publication types, blinding, and the type of design such as parallel or cross over. Crossover trials were evaluated as long as outcome data were available for each treatment segment prior to the crossover.

2.2.2. Type of Participants. This study evaluated retinopathy induced by type 1 and type 2 diabetic mellitus despite of gender, age, or nationality. Diabetic retinopathy could be either nonproliferative diabetic retinopathy (NPDR) or proliferative diabetic retinopathy (PDR). This study excluded participants combined with other ocular diseases like glaucoma or severe cataract. Pregnant and lactating women were also excluded.

2.2.3. Type of Interventions. All forms of radix astragali and its prescriptions intervention regardless of produced pharmaceutical factories were considered as the treatment group. Placebo-controlled, no treatment (blank), or conventional treatment (doxium, neuroprotection, etc.), and other herb treatment (Flos Carthami, etc.) were considered as the comparison group. Laser photocoagulation, acupuncture, or both groups that used radix astragali were excluded.

2.2.4. Type of Outcome Measures. The primary outcomes were visual acuity and fundus manifestations, and secondary outcomes assessed were Fasting Blood Glucose (FBG), HbA1C, TC, TG, erythrocyte sedimentation rate (ESR), plasma viscosity, RBC aggravation index (RAI), and adverse events.

2.3. Data Extraction and Quality Assessment. Data extraction and quality assessment were independently fulfilled by two authors. For each study an author was nominated at random as data extractor, checker, or adjudicator and no one should be data extractor on a paper they authored. Where there was uncertainty regarding eligibility between authors, it was resolved by discussion and consensus or the third party, if necessary. Personal contact was made with authors of published studies when papers contained insufficient information to make a decision about eligibility. Besides looking through abstracts and full-text paper, the authors also paid attention to reference to cull irrelevant citations. The data extraction and quality assessment of all studies were done by two authors following the detailed descriptions of these 


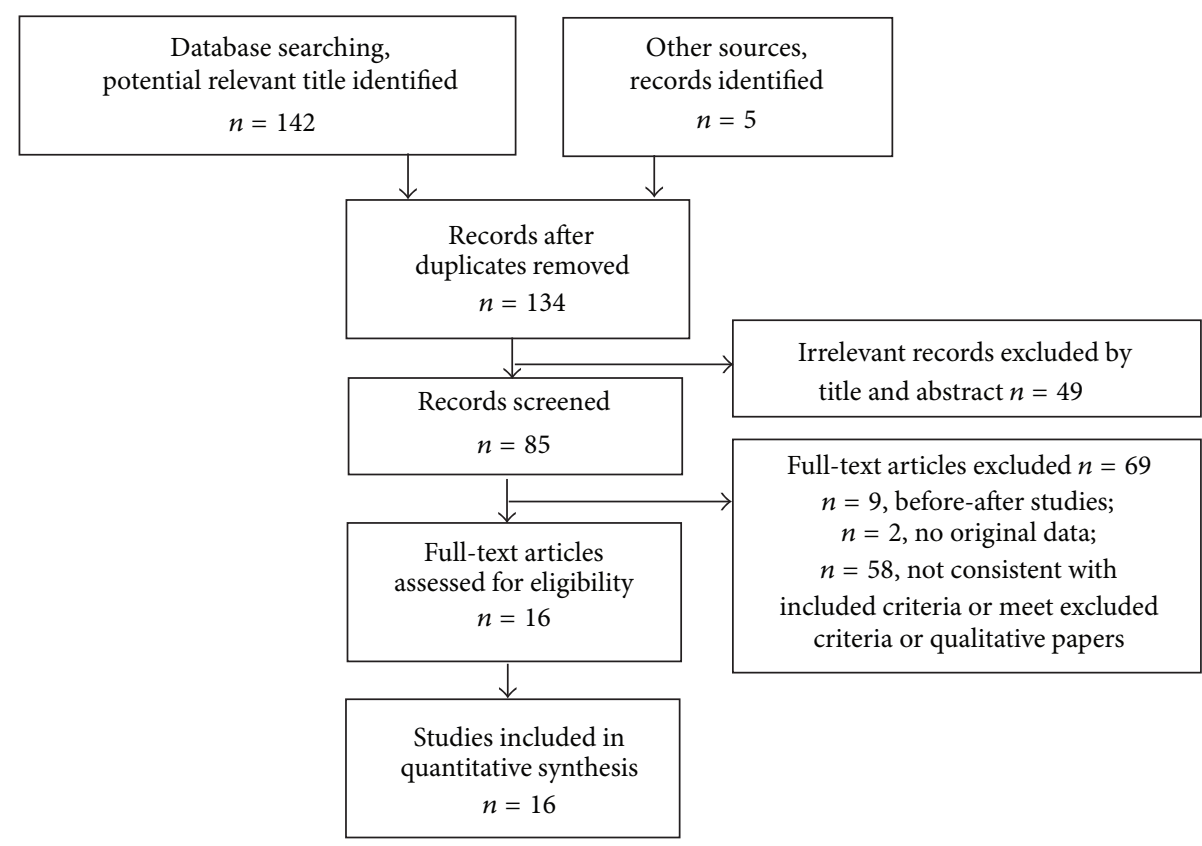

FIGURE 1: Flowchart showing the number of studies evaluated and excluded from the systematic review.

categories as described in Assessing Risk of Bias in the Cochrane Handbook for Systematic Reviews of Interventions by Higgins and Altman [27]. The methodological quality of trials was assessed by the GRADE profiler 3.2.2 software which moved from the evidence to a accommodation for systematic reviews [28] and guidelines in chapter 8 of the Cochrane Handbook for Systematic Reviews of Interventions. And the clinical data were analyzed by RevMan Manager (version 5.0.16 Cochrane Collaboration, Oxford) with the random effect analysis mode. Data analysis followed the guidelines in chapter 9 of interventions. A structured pro forma was used for data extraction (Table 1). According to the type of outcome indexes, measurement data were assessed by weighted mean difference (WMD) and 95\% confidence interval (95\% CI). Before combining data, heterogeneity was estimated by Chi-square test and $I^{2}$ test.

Some studies used decimal point method, that is, Snellen fraction, to value the changes of visual acuity, while the others used five-score method. The authors divided the visual acuity evaluation into two subgroups. One was five-score group; the other was Snellen fraction group. Mean changes from baseline were calculated by posttreatment mean minus pretreatment mean, while $\mathrm{SD}$ (standard deviation) values were estimated by the following formula: $\sqrt{\left|A^{2}-B^{2}\right|+A B}$ ( $A$ : data pretreatment; $B$ : data posttreatment). The final data would round to two decimal places with a decimal place of 0.4 or less got rounded down, while one of 0.5 or more got rounded up.

\section{Results}

3.1. Study Description. A total of 147 studies were initially identified, 142 of them came from electronic database and
5 of them came from other sources. With full-text review, only 16 studies, involving 977 subjects and 1586 eyes (832 eyes in experimental group and 754 eyes in control group) were in accordance with our inclusion criteria and not meet the exclusion criteria. Among the 16 studies, four [30, 36, 37, 44] came from master degree paper database, the rest were journal papers. Figure 1 summarized the search results in flowchart. All trials were conducted in China and published in Chinese.

3.2. Methodological Quality. The quality assessments were summarized in Table 2. Three studies described adequate methods of randomization using a computer-generated randomization [31] or random number tables [30, 36, 44]. The others did not describe the sequence generation process. Except for one study [31], all evaluated trials received allocation scores of "unclear" as they did not have clear descriptions of their method of allocation concealment. Three studies $[30,31,36]$ mentioned blinding; the rest studies were not blinded. One study [31] mentioned loss of participants due to loss of followup. The others had no description of participant dropout. All trials provided patient characteristics in the study group, but lack of data to determine whether these studies had selective outcome reporting or other source of bias. GRADE profiler rated the quality of the evidence low, which indicated that further investigations might influence the confident intervals of this meta-analysis and the result would likely be reversed.

3.3. Characteristics of Included Studies. The characteristics of include studies were shown in Table 1. Among the sixteen studies, all used visual acuity to assess the effect of radix astragali and its prescriptions for diabetic retinopathy, only six reported the adverse events. All the experimental 


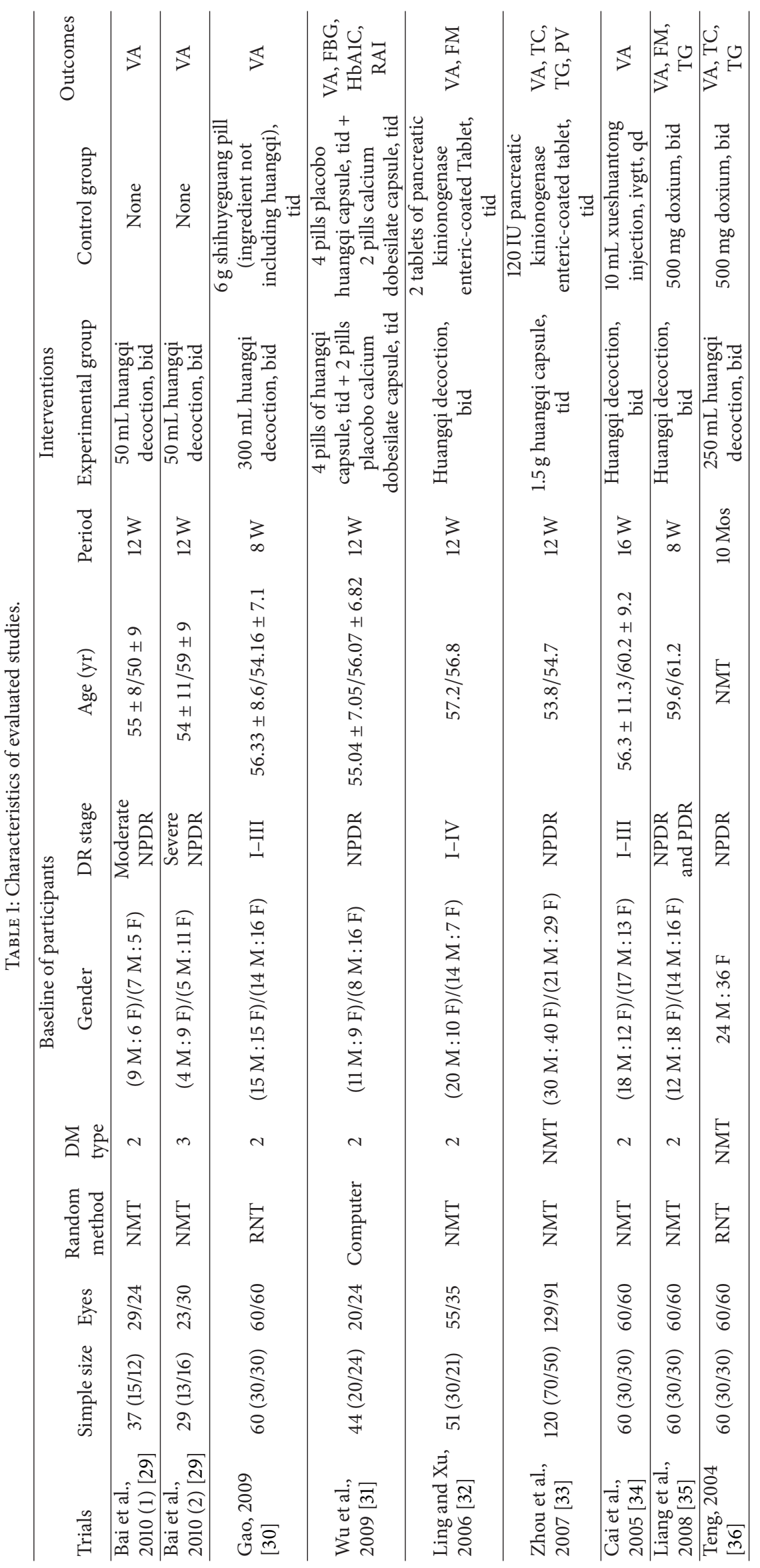




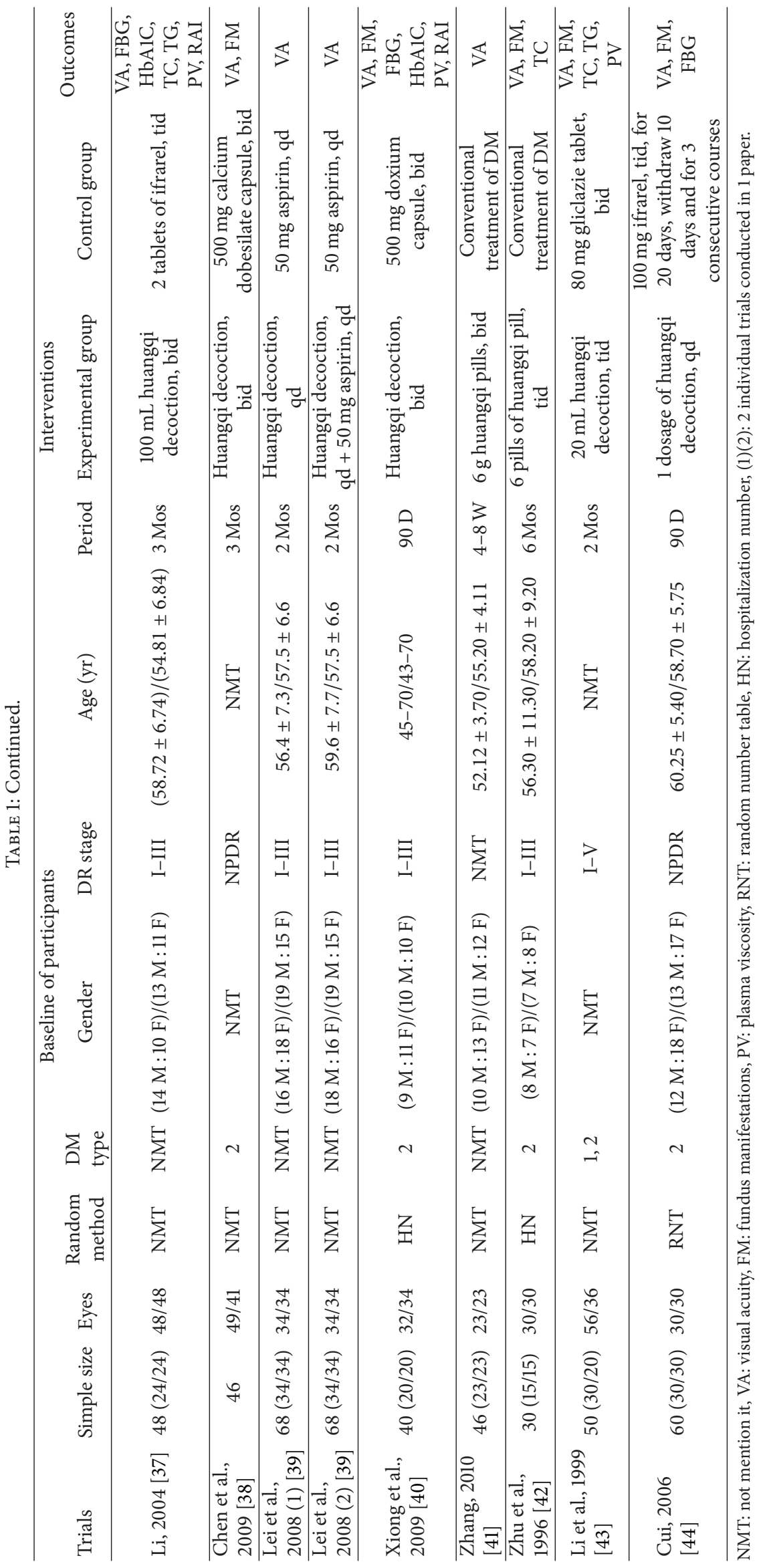




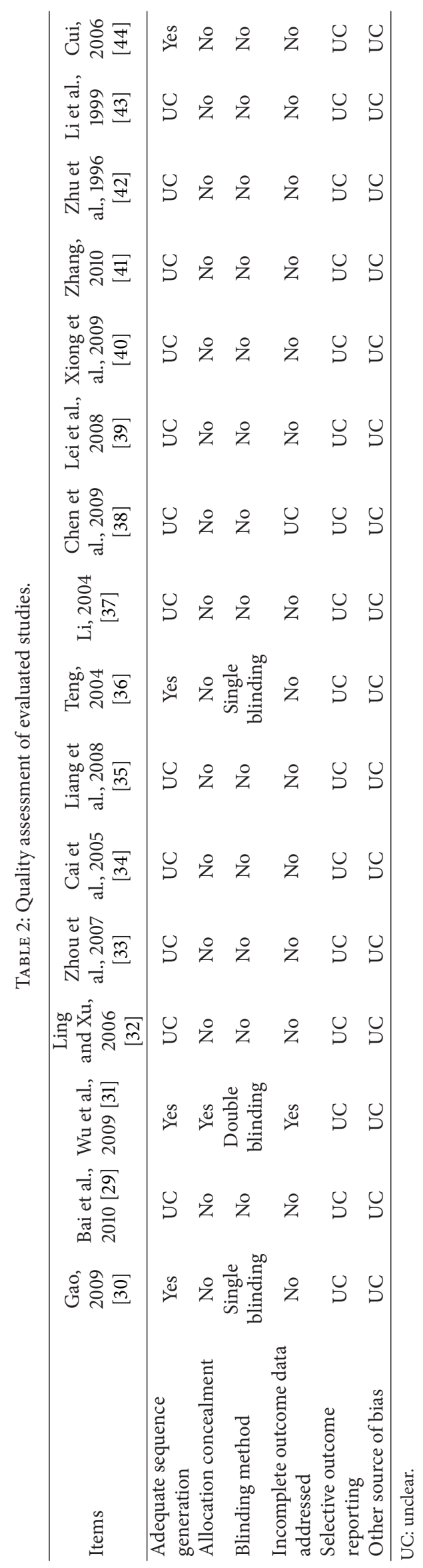




\begin{tabular}{|c|c|c|c|c|c|c|c|c|c|c|c|c|c|}
\hline \multirow{2}{*}{ Study or subgroup } & \multicolumn{3}{|c|}{ Experimental } & \multicolumn{3}{|c|}{ Control } & \multirow{2}{*}{ Weight } & \multirow{2}{*}{$\begin{array}{l}\text { Mean difference } \\
\text { IV, random, } 95 \% \text { CI }\end{array}$} & \multirow[t]{2}{*}{ Year } & \multirow{2}{*}{\multicolumn{4}{|c|}{$\begin{array}{l}\text { Mean difference } \\
\text { IV, random, 95\% CI }\end{array}$}} \\
\hline & Mean & SD & Total & Mean & SD & Total & & & & & & & \\
\hline \multicolumn{14}{|c|}{ 2.1.1 Five scores group } \\
\hline Zhu et al. 1996 & 0.13 & 0.16 & 30 & -0.12 & 0.18 & 30 & $5.6 \%$ & $0.25[0.16,0.34]$ & 1996 & & & - & \\
\hline Li 1999 & 0.11 & 0.13 & 56 & 0.01 & 0.13 & 36 & $5.7 \%$ & $0.1[0.05,0.15]$ & 1999 & & & w & \\
\hline Teng 2004 & 0.21 & 0.23 & 60 & 0.11 & 0.22 & 60 & $5.6 \%$ & $0.1[0.02,0.18]$ & 2004 & & & - & \\
\hline Li 2004 & 0.33 & 0.16 & 48 & 0.08 & 0.18 & 48 & $5.6 \%$ & $0.25[0.18,0.32]$ & 2004 & & & - & \\
\hline Cai et al. 2005 & 0.23 & 0.16 & 60 & 0.08 & 0.18 & 60 & $5.7 \%$ & $0.15[0.09,0.21]$ & 2005 & & & - & \\
\hline Ling and Xu 2006 & 0.27 & 0.12 & 55 & 0.01 & 0.14 & 35 & $5.7 \%$ & $0.26[0.2,0.32]$ & 2006 & & & $m$ & \\
\hline Zhou et al. 2007 & 0.23 & 0.16 & 129 & 0.21 & 0.18 & 91 & $5.7 \%$ & $0.02[-0.03,0.07]$ & 2007 & & & & \\
\hline Gao 2009 & 0.34 & 0.16 & 60 & 0.06 & 0.17 & 60 & $5.7 \%$ & $0.28[0.22,0.34]$ & 2009 & & & $\sigma$ & \\
\hline Zhang 2010 & 1.57 & 0.15 & 23 & 0.36 & 0.12 & 23 & $5.6 \%$ & $1.21[1.13,1.29]$ & 2010 & & & & - \\
\hline Subtotal (95\% CI) & & & 521 & & & 443 & $50.7 \%$ & $0.29[0.09,0.49]$ & & & & & \\
\hline \multirow{2}{*}{\multicolumn{14}{|c|}{$\begin{array}{l}\text { Heterogeneity: } \tau^{2}=0.09 ; \chi^{2}=721.03, \mathrm{df}=8(P<0.00001) ; I^{2}=99 \% \\
\text { Test for overall effect: } Z=2.87(P=0.004)\end{array}$}} \\
\hline & & & & & & & & & & & & & \\
\hline \multicolumn{14}{|c|}{ 2.1.2 Snellen fraction group } \\
\hline Lei et al. 2008(1) & 0.04 & 0.08 & 34 & 0.03 & 0.1 & 34 & $5.7 \%$ & $0.01[-0.03,0.05]$ & & & & & \\
\hline Lei et al. 2008(2) & 0.15 & 0.09 & 34 & 0.03 & 0.1 & 34 & $5.7 \%$ & $0.12[0.07,0.17]$ & & & & - & \\
\hline Bai et al. 2010(2) & 0.12 & 0.18 & 23 & -0.1 & 0.19 & 30 & $5.5 \%$ & $0.22[0.12,0.32]$ & & & & $\rightarrow$ & \\
\hline Cui 2006 & 0.15 & 0.15 & 30 & 0.1 & 0.13 & 30 & $5.6 \%$ & $0.05[-0.02,0.12]$ & 2006 & & & $=$ & \\
\hline Liang et al. 2008 & 0.1 & 0.08 & 60 & 0.04 & 0.11 & 60 & $5.7 \%$ & $0.06[0.03,0.09]$ & 2008 & & & $=$ & \\
\hline Wu et al. 2009 & 0.13 & 0.27 & 20 & 0.02 & 0.33 & 24 & $5 \%$ & $0.11[-0.07,0.29]$ & 2009 & & & - & \\
\hline Xiong et al. 2009 & 0.3 & 0.22 & 32 & 0.17 & 0.23 & 34 & $5.4 \%$ & $0.13[0.02,0.24]$ & 2009 & & & - & \\
\hline Chen et al. 2009 & 0.07 & 0.24 & 49 & 0.03 & 0.41 & 41 & $5.2 \%$ & $0.04[-0.1,0.18]$ & 2009 & & & - & \\
\hline Bai et al. 2010(1) & 0.18 & 0.21 & 29 & -0.01 & 0.18 & 24 & $5.5 \%$ & $0.19[0.08,0.3]$ & 2010 & & & - & \\
\hline Subtotal $(95 \%$ CI $)$ & & & 311 & & & & $49.3 \%$ & $0.09[0.05,0.14]$ & & & & $\downarrow$ & \\
\hline \multicolumn{14}{|c|}{$\begin{array}{l}\text { Heterogeneity: } \tau^{2}=0 ; \chi^{2}=27.79, \mathrm{df}=8(P=0.0005) ; I^{2}=71 \% \\
\text { Test for overall effect: } Z=4.18(P<0.0001)\end{array}$} \\
\hline Total (\%95 CI) & & & 832 & & & 754 & $100 \%$ & $0.2[0.09,0.3]$ & & & & & \\
\hline \multirow{2}{*}{\multicolumn{10}{|c|}{$\begin{array}{l}\text { Heterogeneity: } \tau^{2}=0.05 ; \chi^{2}=863.51, \mathrm{df}=17(P<0.00001) ; I^{2}=98 \% \\
\text { Test for overall effect: } Z=3.66(P=0.0003)\end{array}$}} & -1 & -0.5 & 0.5 & 1 \\
\hline & & & & & & & & & & & $\begin{array}{l}\text { Favours } \\
\text { perimental }\end{array}$ & $\begin{array}{l}\text { Favours } \\
\text { control }\end{array}$ & \\
\hline
\end{tabular}

FIGURE 2: Meta-analysis of visual acuity (radix astragali group versus control group).

interventions were oral administration. The control group intervention included gliclazide, other herbs, placebo, or no treatment. A continuous numerical scale, such as visual acuity, FBG, HbA1C, TC, TG, or plasma viscosity, was used in all include studies. Studies used multiply scales to rate the degrees of visual acuity were not evaluated due to the difficulty to unify standard. Total effects and fundus changes were not pooled because different studies use different standards. So other indexes such as hematocrit (HCT), whole blood viscosity, were not pooled due to the small number of studies and the clinical heterogeneity of these trials. All the participants came from inpatient and/or outpatient department of ophthalmology or endocrinology. The age of participants varied from 43 to 75.75 years. The course of treatment ranged from four weeks to ten months. The experimental interventions comprised huangqi decoctions, pills, and capsules. The control interventions included placebo, various western drugs (e.g.: gliclazide pills), other herbs, and no DR treatment (DM conventional treatment).

\subsection{Outcome Measurements}

3.4.1. Visual Acuity. 16 studies involving 1586 eyes with 832 in experimental group and 754 in control group were synthetized in meta-analysis for visual acuity. The total result in Figure 2 showed that the improvement of visual acuity in radix astragali group was significantly better than that of control group (WMD 0.20, 95\% CI $[0.09,0.30]$ ) with a degree of heterogeneity $\left(X^{2}=863.51, P<0.10, I^{2}=98 \%\right)$. In each subgroup, the incorporated data revealed that visual acuity in radix astragali group was superior to control group (WMD $0.29,95 \%$ CI $[0.09,0.49]$; (WMD 0.09, 95\% CI $[0.05,0.14]$ ) with heterogeneity $\left(X^{2}=721.03, P<0.10, I^{2}=99 \% ; X^{2}=\right.$ 27.79, $\left.P<0.10, I^{2}=71 \%\right)$.

3.4.2. Fundus Manifestations. 7 studies had reported fundus manifestations (Fundus Fluorescein Angiography) involving 578 eyes with 312 in experimental group and 266 in control group. Inefficiency rate was synthetized in the meta-analysis to test effect on fundus manifestations. The result in Figure 3 showed that difference of fundus improvement between radix astragali group and control group was significantly (OR 0.27, 95\% CI $[0.17,0.40], P<0.01)$ with homogeneity $\left(X^{2}=10.38\right.$, $\left.P>0.1, I^{2}=42 \%\right)$.

3.4.3. FBG ( $\mathrm{mmol} / \mathrm{L})$. Only four evaluated studies involving 242 participants reported the reduction of FBG. Metaanalysis shown in Figure 4 suggested that the efficacy of radix astragali and its prescription was superior to the control group (WMD $-0.26,95 \% \mathrm{CI}[-0.51,-0.00]$ ), with no heterogeneity $\left(X^{2}=1.21, P>0.10, I^{2}=0 \%\right)$.

3.4.4. $\mathrm{HbA1C}(\%)$. Three studies indicated that radix astragali group was not better than the control group (WMD 0.45 , $95 \%$ CI $[-1.00,1.90])$ in lower down HbAlC, with a degree 


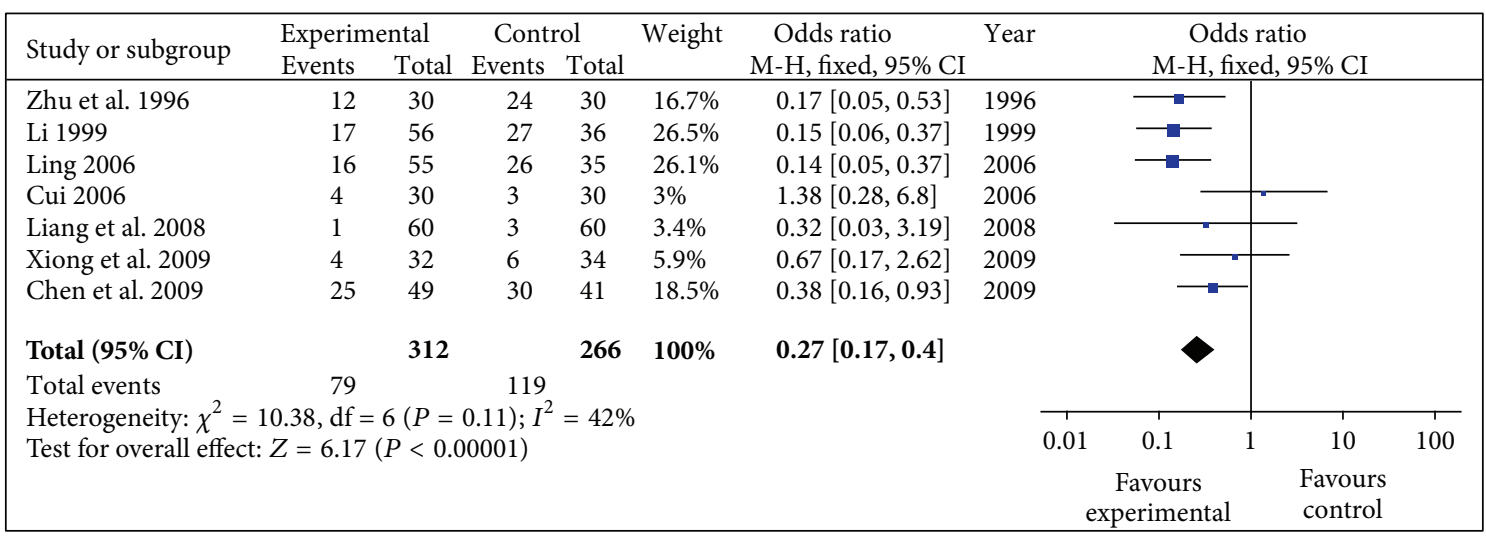

FIGURE 3: Meta-analysis of fundus manifestations (radix astragali group versus control group).

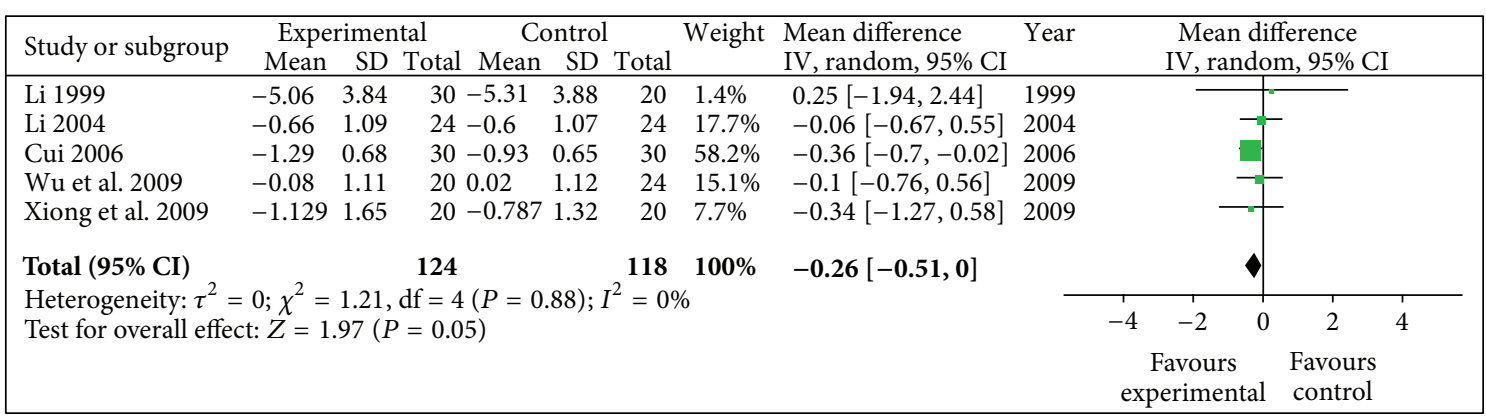

FIGURE 4: Meta-analysis of FBG (radix astragali group versus control group).

of heterogeneity $\left(X^{2}=17.68, P<0.10, I^{2}=89 \%\right)$, shown in Figure 5.

3.4.5. TC $(\mathrm{mmol} / \mathrm{L})$. Reduction of Total Cholesterol in radix astragali group was not significantly better than that of control group (WMD-0.96, 95\% CI [-2.19, 0.27]) with a degree of heterogeneity $\left(X^{2}=209.26, P<0.10, I^{2}=98 \%\right)$, shown in Figure 6.

3.4.6. TG $(\mathrm{mmol} / \mathrm{L})$. Decrease of Triglyceride in radix astragali group was significantly better than that of control group (WMD $-0.36,95 \%$ CI $[-0.60,-0.12]$ ) with heterogeneity $\left(X^{2}=12.74, P<0.10, I^{2}=69 \%\right)$, shown in Figure 7 .

3.4.7. Plasma Viscosity $(\mathrm{mPa} / \mathrm{s})$. Decrease of plasma viscosity in radix astragali group was significantly better than that of control group (WMD $-0.93,95 \%$ CI $[-1.67,-0.20]$ ) with heterogeneity $\left(X^{2}=233.36, P<0.10, I^{2}=99 \%\right)$, shown in Figure 8.

3.4.8. RAI. RAI in radix astragali group was significantly descending compared with control group (WMD $-1.27,95 \%$ CI $[-2.35,-0.19])$ with heterogeneity $\left(X^{2}=41.27, P<0.10\right.$, $\left.I^{2}=95 \%\right)$, shown in Figure 9.

3.5. Publication Bias. Publication bias was assessed using the Begg's rank correlation method. Results are presented as a funnel plot (Figure 10). The funnel plot appeared to be asymmetric. All of the evaluated studies lay within the $95 \%$ $\mathrm{CI}$ and were uniformly distributed around the horizontal line. Since all the evaluated studies were conducted in China and published in Chinese, language bias and geographical bias may result in publication bias.

3.6. Sensitivity Analysis. In the primary analysis, randomeffect models were applied for the analysis of outcome measures of visual acuity, FBG, HbA1C, TC, TG, plasma viscosity and RAI. Only visual acuity had enough studies for making sensitivity. We recalculated the sensitivity analysis (see Figure 11) after removing some low-quality studies [40, 42] for quasi-random method; [41] for its far deviation) and the application of fixed-effect models; it were found that the overall estimates were virtually identical and the 95\% CI were similar between the sensitivity analysis (visual acuity: WMD $0.13,95 \% \mathrm{CI}[0.08,0.18]$ ) and the primary analysis (visual acuity: WMD $0.20,95 \%$ CI $[0.09,0.30])$.

3.7. Adverse Events. We are also interested in investigating any possible adverse events in such studies. Only three papers $[29,31,37]$ reported adverse reactions, but no adverse events had been observed in both groups.

\section{Discussion}

Diabetic retinopathy has a serious impact on patients in terms of visual impairment, and disturbance of glucose, lipid, and 


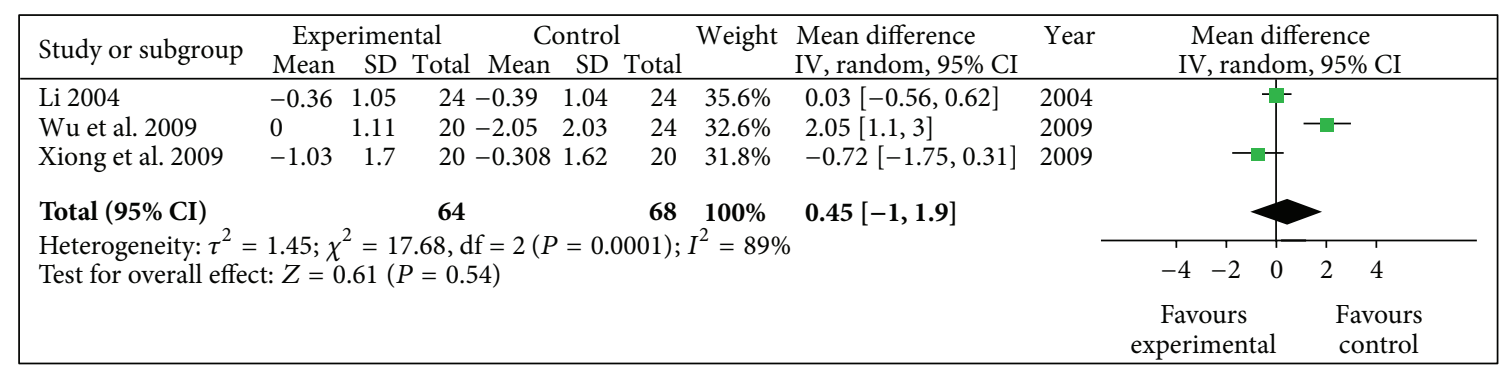

FIgURE 5: Meta-analysis of HbAlC (radix astragali group versus control group).

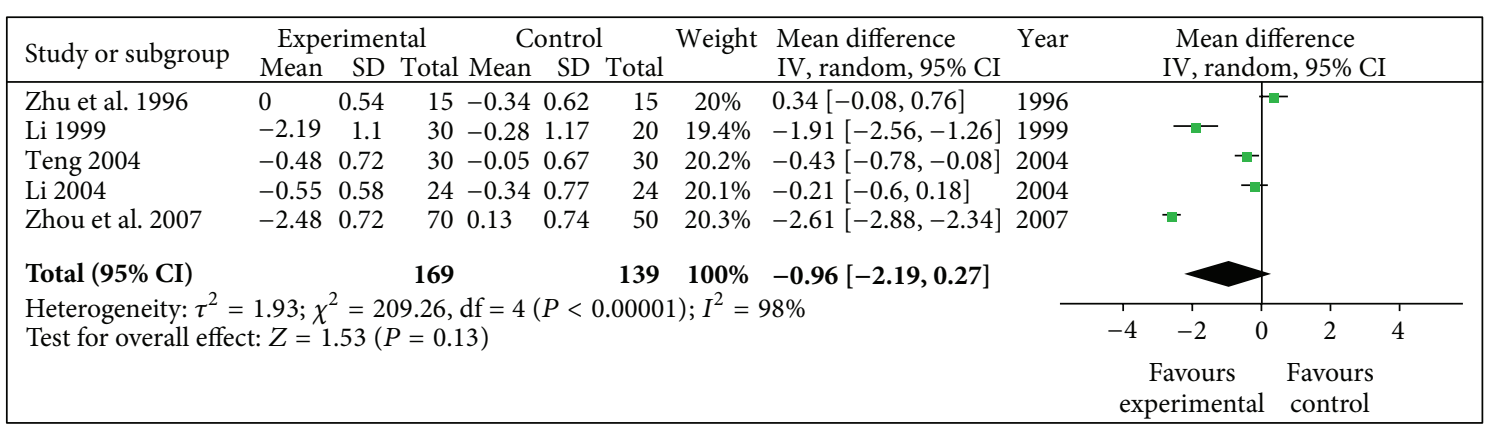

FIgURE 6: Meta-analysis of TC (radix astragali group versus control group).

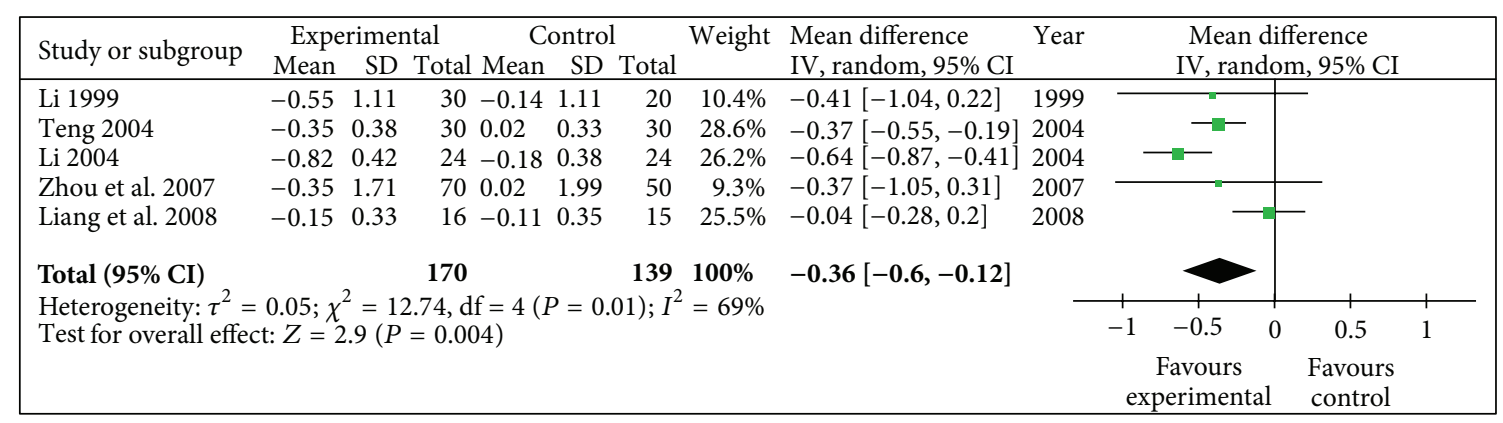

FIGURE 7: Meta-analysis of TG (radix astragali group versus control group).

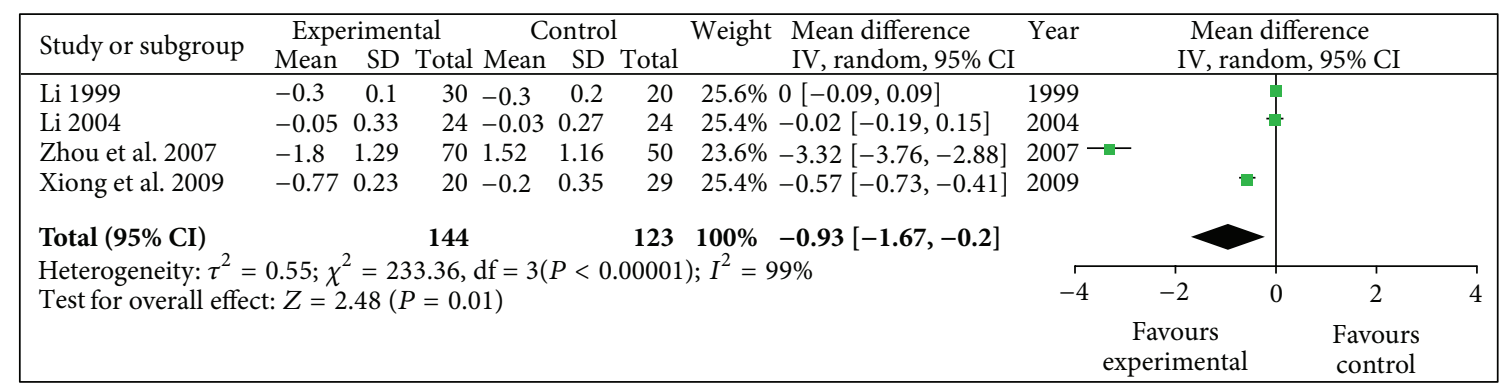

FIGURE 8: Meta-analysis of plasma viscosity (radix astragali group versus control group). 


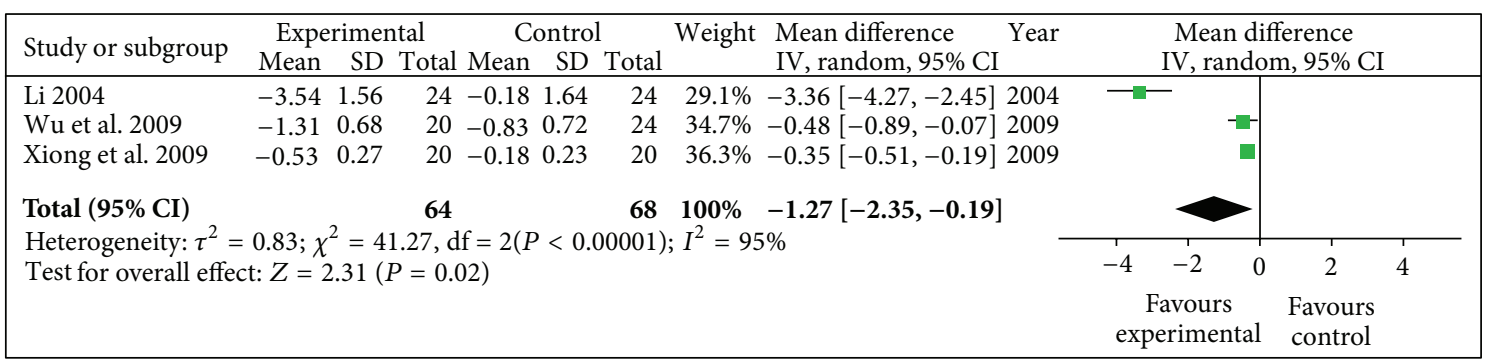

FIGURE 9: Meta-analysis of RAI (radix astragali group versus control group).

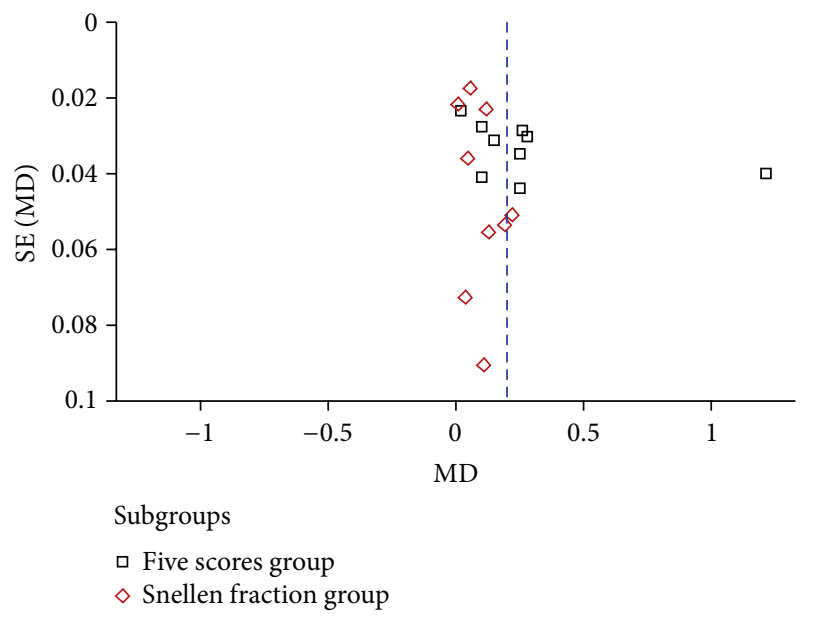

FIgURE 10: Funnel plot of publication bias.

poor quality of life. In many countries, conventional western medicine therapy is considered as the standard treatment for diabetic retinopathy. Most patients are usually treated by improved glycemic control and blood pressure control $[16,45,46]$, calcium dobesilate, anti-VEGF therapy, laser photocoagulation, or vitreoretinal surgeries. However, these managements are far from clinical satisfaction. Taking antiVEGF therapy for example, it is limited by short-lived effects and needs repeated injection and reinforced treatment [4749]. Thereby, it is essential to introduce another effective and safe unconventional treatment of diabetic retinopathy for future drug development. The previous researches revealed that radix astragali was one of the most common used herb for DR. Hence, we have conducted this systematic review to compare the efficacy of radix astragali and its prescriptions with that of conventional western drugs, placebo, or other herbs in the management of DR.

4.1. Result Analysis. In this systematic review, a number of English and Chinese literature databases were searched. We applied a comprehensive search strategy with a language of English and Chinese. Finally, 16 studies involving 977 participants and 1586 eye (832 eyes in experimental group and 754 eyes in control group) were finally identified. The authors incorporated visual acuity, fundus manifestations, FBG, HbA1C, TC, TG, plasma viscosity, and RAI as the outcome measures to perform meta-analysis. The results revealed that, with regard to visual acuity, fundus manifestations, FBG, TG, plasma viscosity, and RAI, the experimental group (radix astragali and its prescriptions) was superior to the control group (placebo, blank, other herbs, and conventional western drugs). While in the perspective of HbA1C and TC, no evidence had been found to support that the experimental group was better than the control group. Moreover, sensitivity analysis of visual acuity of all evaluated studies supported radix astragali and its prescriptions had better effect in improving eyesight. The results showed that radix astragali and its prescriptions had some potential as future therapeutic targets in DR; the mechanism might lay on reducing FBG, TG, plasma viscosity and RAI to perform the beneficial function of improving visual acuity. Although it showed no favour of reducing $\mathrm{HbA1C}$ and TC, it might possibly attributed to small evaluated literatures and observing time. Plus, methodological quality assessment suggested the evidence was low, so the results should be read with care.

4.2. Limitations of This Study. In general, the evidence of radix astragali and its prescriptions for diabetic retinopathy is positive. These data suggested that radix astragali and its prescriptions alleviated diabetic retinopathy in the perspective of improving visual acuity and fundus manifestations, reducing FBG, TG, RAI, and plasma viscosity, which seemed to be superior to control group. However, these results seem to be misread and should be carefully explained, due to following factors. (1) Design of studies: these clinical trials seldom provided details of their randomized techniques (except three studies) and clear statement of allocation concealment (except one study). In addition, only one study described loss of data, most of the studies not mentioned the followup length. Therefore, the significant difference between the experimental group and the control group might be a result of low quality of methodologies. (2) Blinding of studies: this study had a limitation in that it had been obvious to investigators that which group was being tested for its efficacy, which group's treatment was merely an ordinary or conventional treatment. Only one study [31] mentioned the double blinding, two studies $[30,36]$ mentioned the single blinding, the rest had no description on blinding. Consequently, the experimental group, having read about the purpose of the study and about the interventions, must have had heightened expectation relative to the control group. 


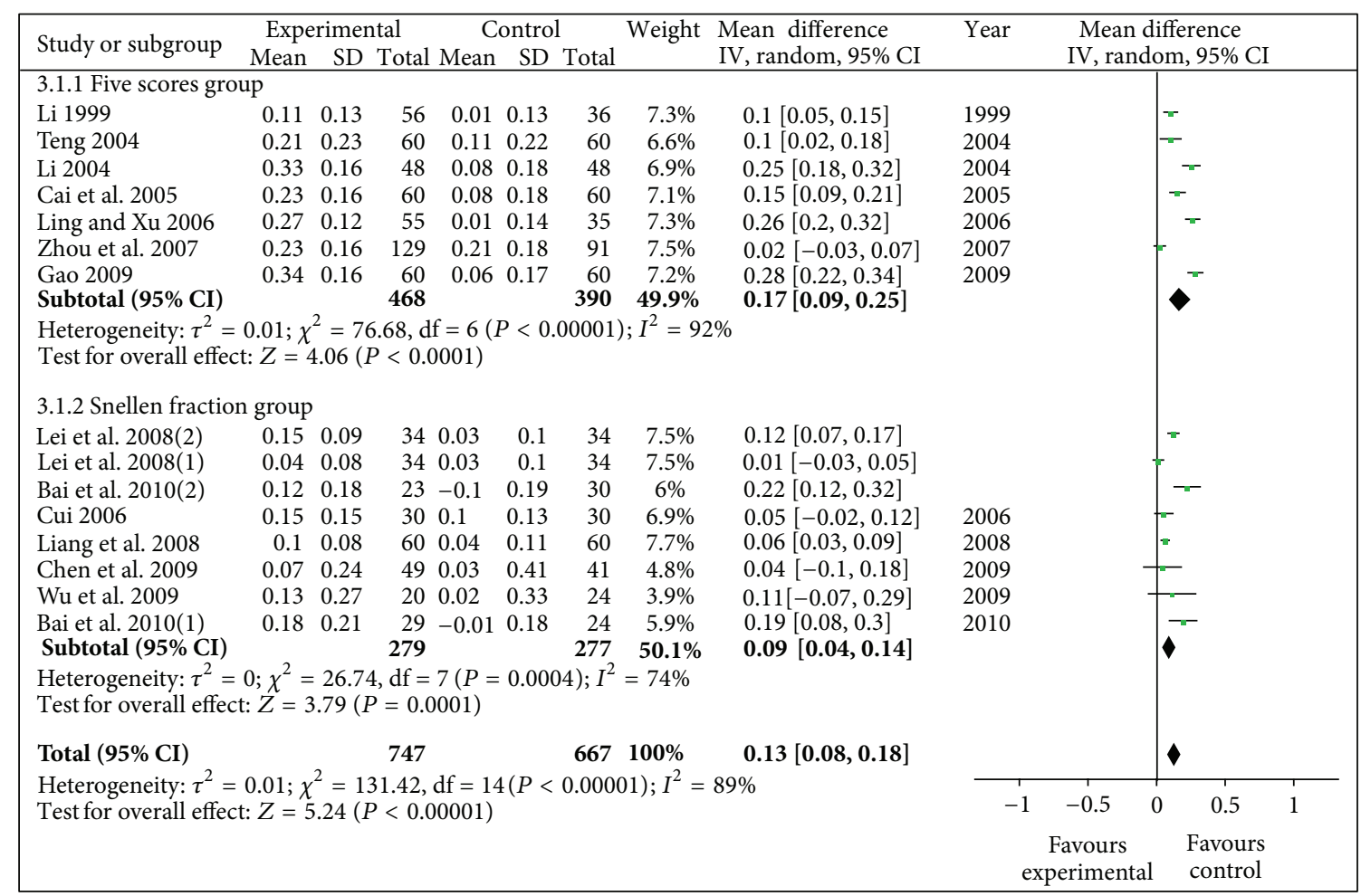

FIGURE 11: Sensitivity analysis of visual acuity (radix astragali group versus control group, 13 studies evaluated).

Therefore, there seemed to have relative potential bias with regard to investigators or participants. (3) Characteristics of participants: variance among age, basic vision, different type of DM, treating course in these studies were too great to have no bias in baseline. It is also hard for authors to identify diabetic macular edema (DME) in evaluated patients, because DME is very important factor that will have a dramatic effect on changes of visual acuity. If the patient has DME, the visual acuity in radix astragali group may be overestimated after treatment while the visual acuity in control group will abirritate the effect of radix astragali. The authors have thought of dividing patients into PDR and NPDR subgroups. However, the data were not reported in the evaluated studies. (4) Publication bias: the funnel plot detected publication bias. It may lie in the evaluated studies which were all conducted in China and published in Chinese. So this study was limited to national health boundaries regarding leaking out-of-area data.

Besides, systematic reviews are secondary research ("research on research"). The object of scrutiny is not the effect of radix astragali and its prescriptions for diabetic retinopathy, but the literatures on this topic. So while this type of research cannot be used to "prove" a hypothesis, the compiled data can encourage the generation of new hypotheses that can then be tested prospectively, with new data. So, systematic reviews are best suited to hypothesis-generation. Besides, comparisons in systematic reviews should be planned, based on directional hypotheses, and limited to a reasonable number of studies. Consequently, the author strongly recommended researchers should firstly come to a consensus about the most appropriate specific and defined protocol for this type of study and use the same protocol for future studies.

On the other hand, the methodological quality of evaluated studies in this paper was generally low. To improve the quality, future researchers should report the method of randomization and allocation concealment, use binding as far as possible. To further verify the efficacy and safety as to obtain the best evidence outside, larger, multicenter clinical studies with prolonged follow-up time are urgently desired.

\section{Conclusion}

The combined results showed that radix astragali and its prescriptions had positive effect to improve DR patients' visual acuity and fundus manifestations and to reduce FBG, TG, plasma viscosity, and RAI. At the same time, the result also showed that current evidence could not prove astragalus preparation treatment group was better than control group in regulating $\mathrm{HbAlC}$ and TC. Radix astragali and its prescriptions had shown some potential as future therapeutic targets in DR; however, the evidence was not sufficient due to low quality of all included studies. Thereby well-designed, largescale high-quality randomized controlled trials are warranted for stronger evidence. And information on adverse effects should also be provided in future trials.

\section{Authors' Contribution}

L. Cheng completed the design of the protocol and wrote the draft of the paper. L. Cheng and G. Zhang performed 
the literature search, data extraction, and meta-analysis. Y. Zhou helped with data extraction, and data analysis. X. Lu, F. Zhang, and H. Ye were taken as a third party to judge the uncertainty. J. Duan participated in the design of the protocol and gave statistical suggestions. All the above authors contributed to the final paper.

\section{Conflict of Interests}

No competing financial interests existed.

\section{Acknowledgments}

The authors acknowledge Honglin Chen who performed preliminary systematic review of radix astragali for diabetic retinopathy four years ago as supplying additional information for us. In addition, they are thankful for funding support of national "11th five-year" plan key technology R\&D program (no. 2006BAI04A0401) and Chinese Scholarship Council (CSC).

\section{References}

[1] G. D. Rogell, "Late vitreous haemorrhage after successful diabetic pan retinal photocoagulation," in Proceedings of the American Academy of Ophthalmology Annual Meeting, Dallas, Tex, USA, 1987.

[2] H. Lewis, G. W. Abrams, M. S. Blumenkranz, and R. V. Campo, "Vitrectomy for diabetic macular traction and edema associated with posterior hyaloidal traction," Ophthalmology, vol. 99, no. 5, pp. 753-759, 1992.

[3] S. Charles and C. E. Flinn, "The natural history of diabetic extramacular traction retinal detachment," Archives of Ophthalmology, vol. 99, no. 1, pp. 66-68, 1981.

[4] Group DRVSR, "Early vitrectomy for severe vitreous hemorrhage in diabetic retinopathy. Two-year results of a randomized trial. diabetic retinopathy vitrectomy study report 2 . The diabetic retinopathy vitrectomy study research group," Archives of Ophthalmology, vol. 103, no. 11, pp. 1644-1652, 1985.

[5] M. D. Davis, "Vitreous contraction in proliferative diabetic retinopathy," Archives of Ophthalmology, vol. 74, no. 6, pp. 741751, 1965.

[6] Cochrane Library, "MeSH Definition," 2011, http://onlinelibrary .wiley.com/cochranelibrary/search/mesh/quick.

[7] D. Vaughan, T. Asbury, and P. Riordan-Eva, General Ophthalmology, People's Medical Publishing House, Beijing, China, 15th edition, 2003.

[8] Y. H. Cho, M. E. Craig, S. Hing et al., "Microvascular complications assessment in adolescents with 2- to 5-yr duration of type 1 diabetes from 1990 to 2006," Pediatric Diabetes, vol. 12, no. 8 , pp. 682-689, 2011.

[9] A. K. Sjølie, P. Dodson, and F. R. R. Hobbs, "Does reninangiotensin system blockade have a role in preventing diabetic retinopathy? A clinical review," International Journal of Clinical Practice, vol. 65, no. 2, pp. 148-153, 2011.

[10] S. Sivaprasad, B. Gupta, R. Crosby-Nwaobi, and J. Evans, "Prevalence of diabetic retinopathy in various ethnic groups: a worldwide perspective," Survey of Ophthalmology, vol. 57, no. 4, pp. 347-370, 2012.
[11] Y. Alvarez, K. Chen, A. L. Reynolds, N. Waghorne, J. J. O'Connor, and B. N. Kennedy, "Predominant cone photoreceptor dysfunction in a hyperglycaemic model of non-proliferative diabetic retinopathy," Disease Models and Mechanisms, vol. 3, no. 3-4, pp. 236-245, 2010.

[12] J. F. Arevalo and R. A. Garcia-Amaris, "Intravitreal Bevacizumab for diabetic retinopathy," Current Diabetes Reviews, vol. 5, no. 1, pp. 39-46, 2009.

[13] N. Cheung, P. Mitchell, and T. Y. Wong, "Diabetic retinopathy," The Lancet, vol. 376, no. 9735, pp. 124-136, 2010.

[14] Y. Zheng, M. He, and N. Congdon, "The worldwide epidemic of diabetic retinopathy," Indian Journal of Ophthalmology, vol. 60, no. 5, pp. 428-431, 2012.

[15] E. Y. Chew, W. T. Ambrosius, M. D. Davis et al., "Effects of medical therapies on retinopathy progression in type 2 diabetes," The New England Journal of Medicine, vol. 363, no. 3, pp. 233-244, 2010.

[16] F. Ismail-Beigi, T. Craven, M. A. Banerji et al., "Effect of intensive treatment of hyperglycaemia on microvascular outcomes in type 2 diabetes: an analysis of the ACCORD randomised trial," The Lancet, vol. 376, no. 9739, pp. 419-430, 2010.

[17] A. K. Dubey, P. N. Nagpal, S. Chawla, and B. Dubey, "A proposed new classification for diabetic retinopathy: the concept of primary and secondary vitreopathy," Indian Journal of Ophthalmology, vol. 56, no. 1, pp. 23-29, 2008.

[18] M. Malek, M. E. Khamseh, R. Aghili, Z. Emami, L. Najafi, and H. R. Baradaran, "Medical management of diabetic retinopathy: an overview," Archives of Iranian Medicine, vol. 15, no. 10, pp. 635640, 2012.

[19] J. F. Arevalo, J. G. Sanchez, A. F. Lasave et al., "Intravitreal Bevacizumab (Avastin) for diabetic retinopathy at 24-months: the 2008 Juan Verdaguer-Planas lecture," Current Diabetes Reviews, vol. 6, no. 5, pp. 313-322, 2010.

[20] P. Iacono, M. B. Parodi, and F. Bandello, "Antivascular endothelial growth factor in diabetic retinopathy," Developments in Ophthalmology, vol. 46, pp. 39-53, 2010.

[21] J. F. Arevalo, J. G. Sanchez, A. F. Lasave et al., "Intravitreal bevacizumab (Avastin) for diabetic retinopathy: the 2010 GLADAOF lecture," Journal of Ophthalmology, vol. 2011, Article ID 584238, 13 pages, 2011.

[22] T. Yasukawa, Y. Tabata, H. Kimura, and Y. Ogura, "Recent advances in intraocular drug delivery systems," Recent Patents on Drug Delivery and Formulation, vol. 5, no. 1, pp. 1-10, 2011.

[23] W. L. Li, H. C. Zheng, J. Bukuru, and N. de Kimpe, "Natural medicines used in the traditional Chinese medical system for therapy of diabetes mellitus," Journal of Ethnopharmacology, vol. 92, no. 1, pp. 1-21, 2004.

[24] H. Zhang, C. G. Tan, H. Z. Wang, S. B. Xue, and M. Q. Wang, "Study on the history of traditional Chinese medicine to treat diabetes," European Journal of Integrative Medicine, vol. 2, no. 1, pp. 41-46, 2010.

[25] W. Xie and L. Du, "Diabetes is an inflammatory disease: evidence from traditional Chinese medicines," Diabetes, Obesity and Metabolism, vol. 13, no. 4, pp. 289-301, 2011.

[26] W. Jia, W. Y. Gao, and P. G. Xiao, "Antidiabetic drugs of plant origin used in China: compositions, pharmacology, and hypoglycemic mechanisms," China Journal of Chinese Materia Medica, vol. 28, no. 2, pp. 108-113, 2003.

[27] J. P. T. Higgins and D. G. Altman, "Assessing risk of bias in included studies," in Cochrane Handbook for Systematic Reviews of Interventions Version 5.0.1 (Updated September 2008), J. 
P. T. Higgins and S. Green, Eds., chapter 8, The Cochrane Collaboration, http://www.cochrane-handbook.org/.

[28] G. H. Guyatt, A. D. Oxman, H. J. Schünemann, P. Tugwell, and A. Knottnerus, "GRADE guidelines: a new series of articles in the Journal of Clinical Epidemiology," Journal of Clinical Epidemiology, vol. 64, no. 4, pp. 380-382, 2011.

[29] Q. Bai, G. C. Wei, and B. Zhang, "Zhixuesanyumingmu decoction in non-proliferative diabetic retinopathy," Chinese General Practice, vol. 13, no. 15, pp. 1642-1645, 2010.

[30] H. Gao, The Theory of Xuanfu and Its Application of Diabetic Retinopathy, Hebei Medical University, 2009.

[31] L. Wu, F. Yan, H. Su, and L. Jian, "Clinical study on Qihuang Mingmu granule for the treatment of diabetic retinopathy (blood stasis and deficiency of qi and yin)," China Journal of Chinese Ophthalmology, vol. 19, no. 2, pp. 74-78, 2009.

[32] Q. Ling and D. Y. Xu, "A summary on 30 cases of retinopathy complicated by type 2 diabetes treated by yiqiyangyinhuoxue decoction," Hunan Journal of Traditional Chinese Medicine, vol. 22, no. 5, pp. 8-9, 2006.

[33] M. Zhou, H. P. Mi, Z. W. Guo, and L. X. Bian, "Clinical analysis of 70 cases of diabetic retinopathy by Tangwang capsule," Liaoning Journal of Traditional Chinese Medicine, vol. 34, no. 4, pp. 457-458, 2007.

[34] G. H. Cai, G. Li, X. Y. Hong, Q. C. Chen, and N. R. Li, "Clinical observation of 30 cases of diabetic retinopathy by integrated Chinese and western medicine," New Journal of Traditional Chinese Medicine, vol. 37, no. 8, pp. 63-64, 2005.

[35] F. M. Liang, L. Wang, X. Y. Xie, H. Z. Shao, and W. J. Liu, "Clinical study on treatment of diabetic retinal neovascularization by, 'Yiqi Quyu Huatan decoction,' Shanghai Journal of Traditional Chinese Medicine, vol. 42, no. 7, pp. 25-27, 2008.

[36] X. M. Teng, The Clinical Study on the Treatment of Huayumingmutang to Diabetic Retinopathy of Non-Proliferative Phase, Heilongjiang University of TCM, 2004.

[37] D. Q. Li, Clinical Study on Tangwangming Decoction for Pure Diabetic Retinopathy, Beijing University of TCM, 2004.

[38] C. Chen, Y. Q. Zhang, and J. S. Gao, "Therapeutic effect observation of Tangmuning on early diabetic retinopathy," China Journal of Chinese Ophthalmology, vol. 19, no. 2, pp. 7981, 2009.

[39] C. X. Lei, R. M. Li, and Z. H. Wu, "Study of integrated traditional Chinese and western medicine on diabetic retinopathy," Modern Journal of Integrated Traditional Chinese and Western Medicine, vol. 17, no. 11, pp. 1631-1633, 2008.

[40] J. Xiong, Q. H. Peng, Q. L. Wu, J. S. Yu, and Y. H. Fan, "Observation on Yiqi Yangyin and Huoxue Lishui method of treating diabetic retinopathy of qi and yin deficiency and blood obstruction type," China Journal of Chinese Ophthalmology, vol. 12, no. 6, pp. 311-315, 2009.

[41] Z. H. Zhang, "23 cases of diabetic retinopathy treated by Pingxiaobaoshi pills," Journal of Emergency in Traditional Chinese Medicine, vol. 19, no. 6, pp. 949-950, 2010.

[42] B. Zhu, L. P. Qi, Y. Z. Tang, and L. Z. Lu, "Preliminary clinical observation on the treatment of non-proliferative diabetic retinopathy with mingmu capsula no. 1," China Journal of Chinese Ophthalmology, vol. 6, no. 2, pp. 90-92, 1996.

[43] Y. A. Li, Q. H. Qian, and Y. C. Cheng, "Clinical observation of diabetic retinopathy by compounded Chinese medicine," Journal of Nanjing University of TCM, vol. 15, no. 5, pp. 279-281, 1999.
[44] S. L. Cui, The Clinical Observation on the Treatment of Shenhua Ultra Micro Decoction Piece on Patients with the Pure Diabetic Retinopathy of Qi and Yin Deficiency and Blood Obstructed Type, Hunan University of TCM, 2006.

[45] H. N. Ginsberg, M. B. Elam, L. C. Lovato et al., "Effects of combination lipid therapy in type 2 diabetes mellitus," The New England Journal of Medicine, vol. 362, no. 17, pp. 1563-1574, 2010.

[46] W. C. Cushman, G. W. Evans, R. P. Byington et al., "Effects of intensive blood-pressure control in type 2 diabetes mellitus," The New England Journal of Medicine, vol. 362, no. 17, pp. 1575$1585,2010$.

[47] E. J. Chung, S. J. Kang, J. S. Koo, Y. J. Choi, H. E. Grossniklaus, and H. J. Koh, "Effect of intravitreal bevacizumab on vascular endothelial growth factor expression in patients with proliferative diabetic retinopathy," Yonsei Medical Journal, vol. 52, no. 1, pp. 151-157, 2011.

[48] S. Tatlipinar, U. A. Dinc, N. M. Yenerel, and E. Gorgun, "Shortterm effects of a single intravitreal bevacizumab injection on retinal vessel calibre," Clinical and Experimental Optometry, vol. 95, no. 1, pp. 94-98, 2012.

[49] S. Rangasamy, P. G. McGuire, and A. Das, "Diabetic retinopathy and inflammation: novel therapeutic targets," Middle East African Journal of Ophthalmology, vol. 19, no. 1, pp. 52-59, 2012. 


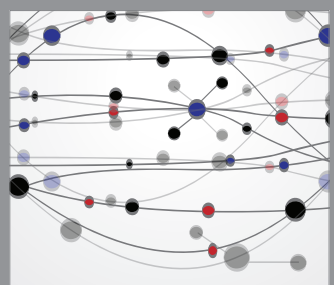

The Scientific World Journal
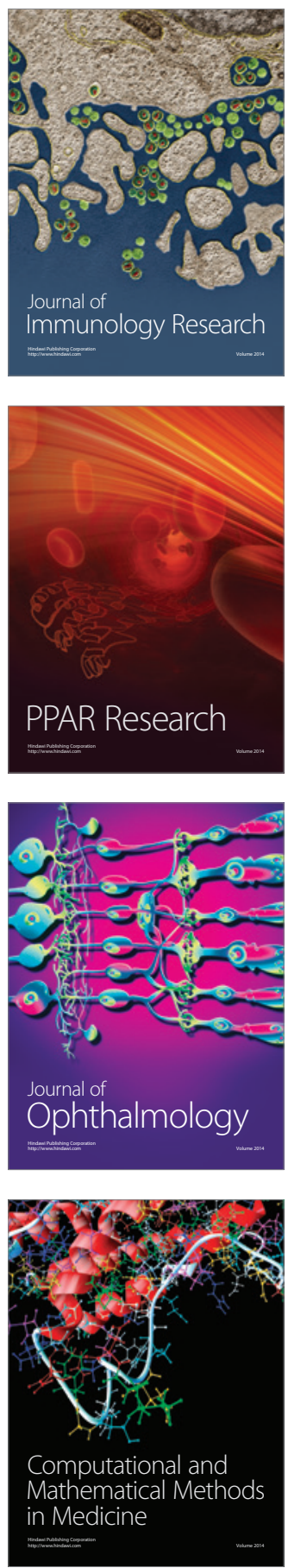

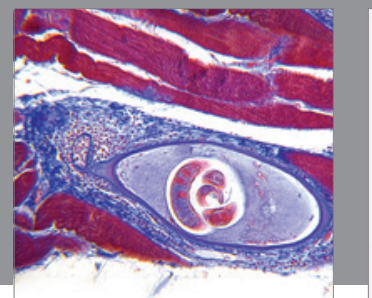

Gastroenterology

Research and Practice
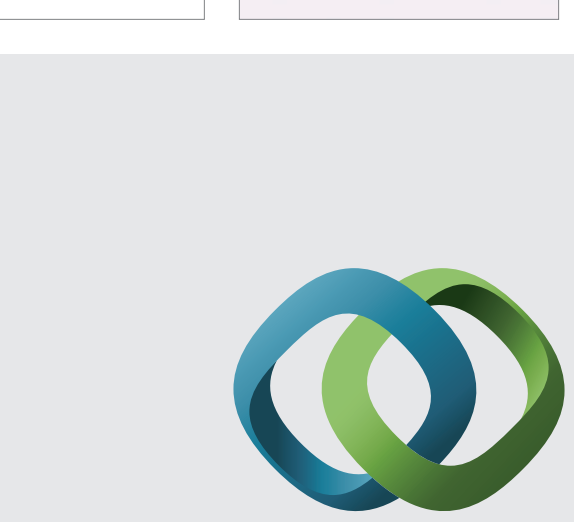

\section{Hindawi}

Submit your manuscripts at

http://www.hindawi.com
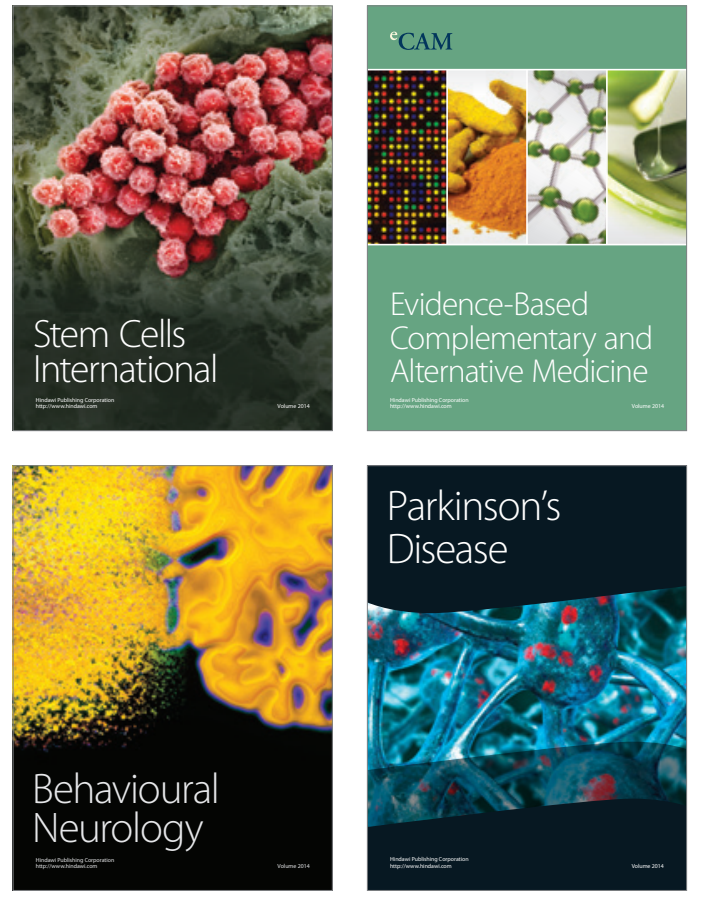
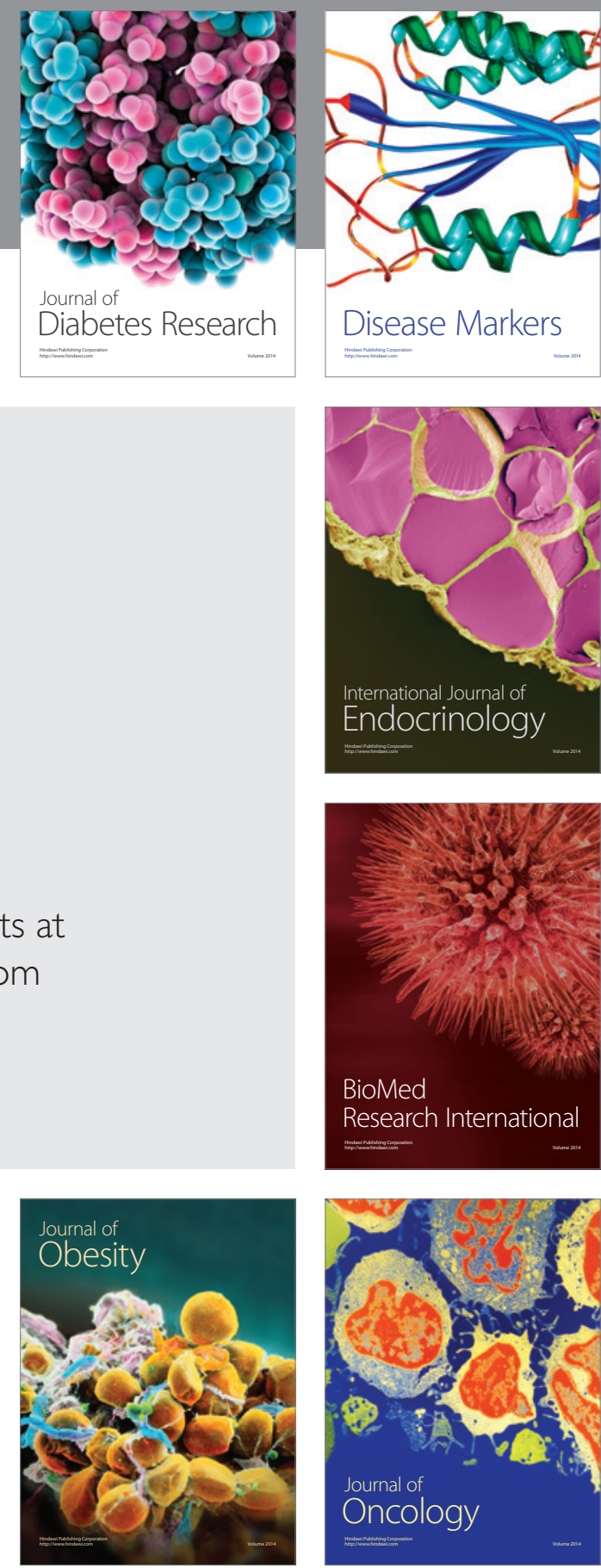

Disease Markers
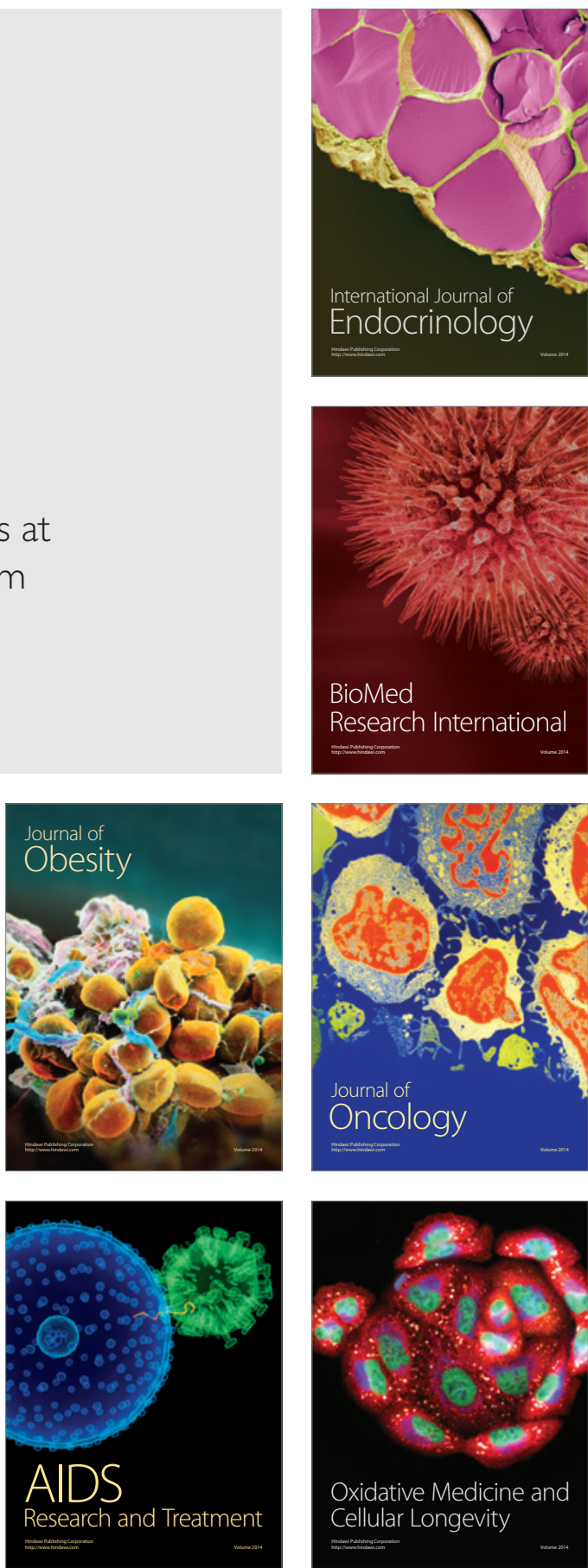